\title{
The Characterization of the Effect of Electric Fields on Tobacco Mosaic Virus (TMV) Alignment using Atomic Force Microscopy (AFM)
}

\author{
E. Widjaja, D. J. Eckerle, J. T. Sloan, and M. T. Harris \\ Purdue University, School of Chemical Engineering, West Lafayette, IN 47906
}

Wild-type TMV is a well studied biological molecule. It has a cylindrical shape with a diameter of $18-20 \mathrm{~nm}$ and a length of $300 \mathrm{~nm}$ with a $4 \mathrm{~nm}$-wide inner channel. The internal and external surfaces of TMV contain charged amino acids, which may serve as potential sites for materials deposition. Researchers have shown that TMV is a suitable biotemplate for nanotubes and nanowires construction. Silica, gold, silver, and other metal clusters have been deposited onto wild-type and genetically engineered tobacco mosaic virus [1, 2].

The purified wild-type TMV is stored in buffer solution, material deposition reactions also occur in solution. For the realization of useful nanotubes, nanowires and electronic components, it is necessary to remove the solvent resulting in the deposition of dried TMV particles on a solid surface. Deposition of these nanorods with a controlled position and orientation is desirable. There are a variety of methods that can be utilized in order to deposit and to align anisotropic particles on a solid substrate such as dip coating and drop evaporation methods [3, 4]. These methods had been used because the hydrodynamics create a shear flow adjacent to the surface to help align the particles.

In this work, drop evaporation and dip coating methods have been used to deposit TMV onto a silicon wafer in the absence and presence of an electric field. It is convenient to use AFM to characterize the deposition and the alignment of TMV. A ring shape of particles deposit was obtained after drop evaporation. Most of TMV particles were found to be on the ring area. Transmitted Electron Microscope (TEM) was used to image TMV which align its long side parallel to the perimeter of the ring. On the area inside the ring TMV particles were found to be in random alignment. TMV particles with random orientation were also found on the surface of the dip coated plate. The random orientation was due to the dominance of Brownian forces acting on the particles over the shear force. To increase the attractive force between the particles and the substrate and to increase alignment by overcoming the Brownian forces, we are interested in imposing electric field during drop evaporation and dip coating experiments.

\section{References}

[1] W. Shenton et al., Adv. Mater. 11(1999) 253.

[2] S. Y. Lee et al., Nanotechnology 16(2005) S435.

[3] M. Chopra et al., J. Rheol. 47(2003) 1111.

[4] M. E. Spotniz et al., J. Mater. Chem. 14(2004) 1299.

[5] This research is supported by Department of Energy office of basic energy Science and Purdue Summer Undergraduate Research Fellowships (SURF) program. 


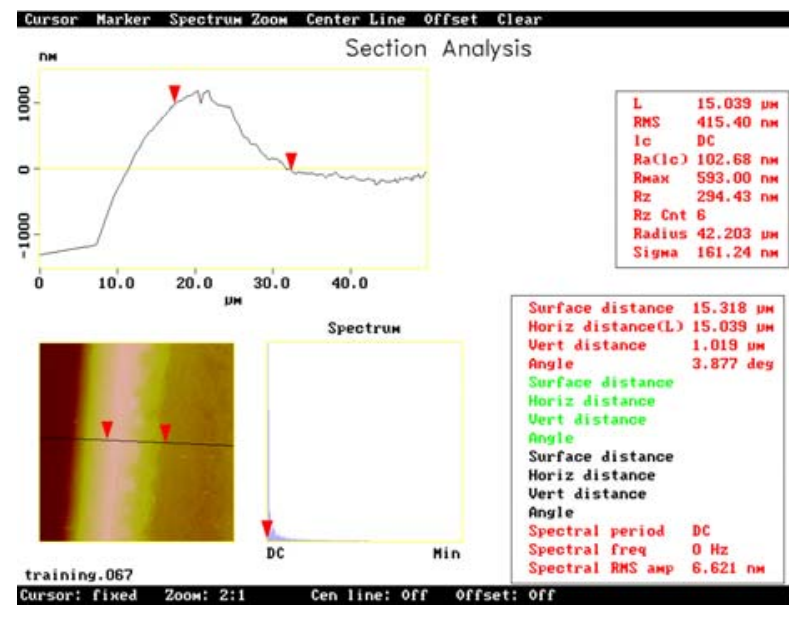

(a)

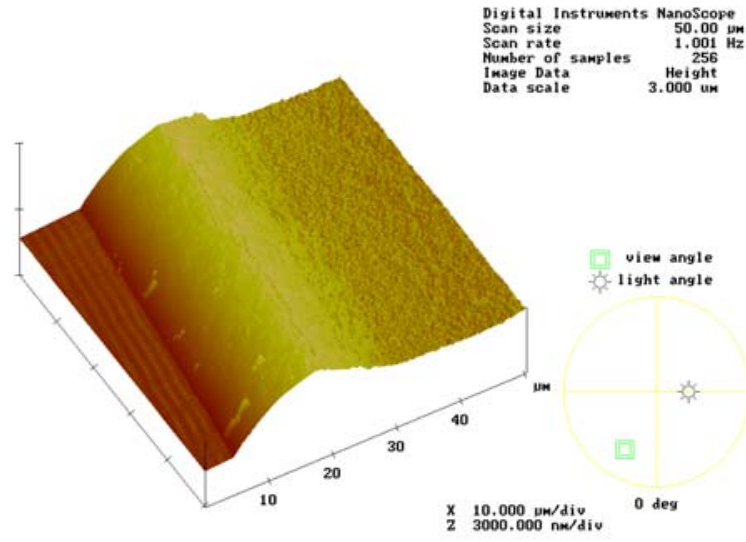

(b)

FIG. 1. AFM image of the edge of the ring after evaporation of a drop of $0.2 \mathrm{mg} / \mathrm{ml} \mathrm{TMV} \mathrm{in} \mathrm{H}_{2} \mathrm{O}$, (a) 2-d image from the side and from the top, and (b) 3-d image from the top

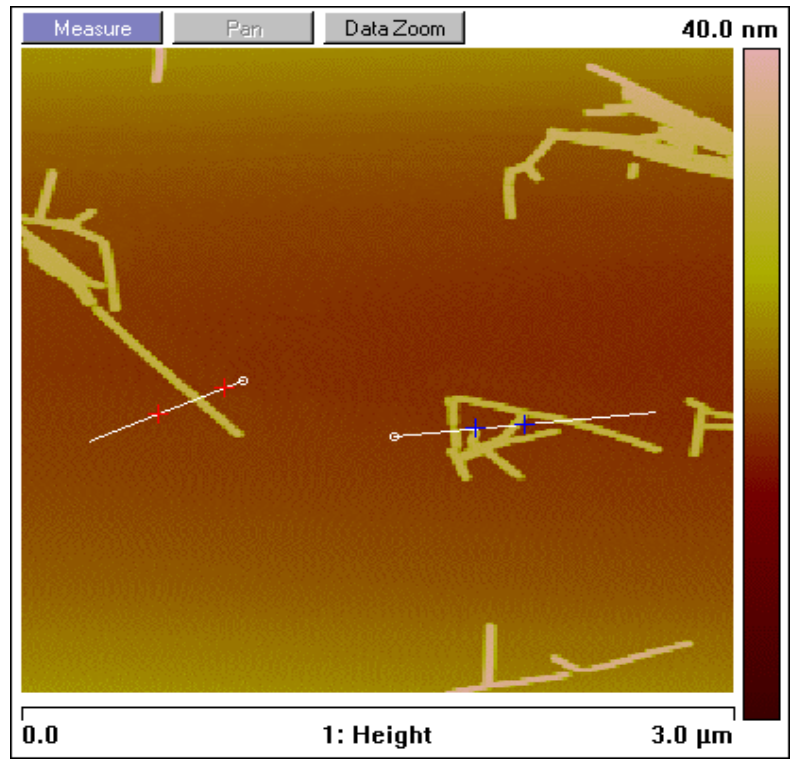

(a)

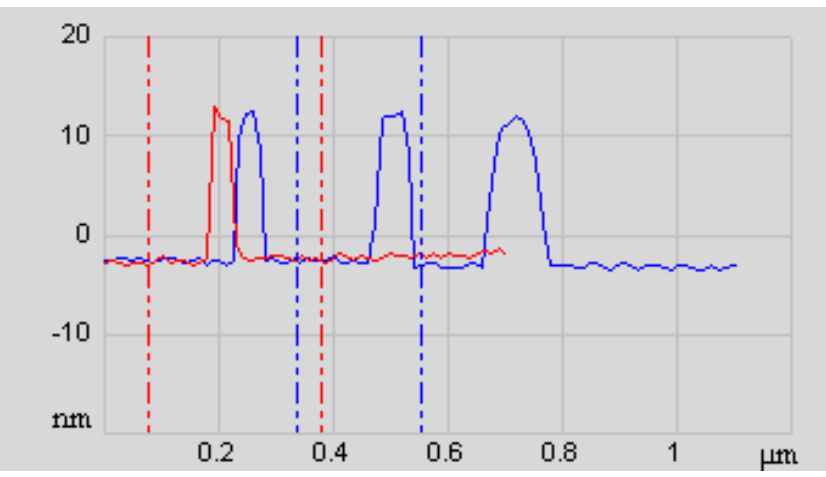

(b)

FIG. 2. AFM image of silicon substrate surface dip-coated in $0.05 \mathrm{mg} / \mathrm{mL}$ TMV solution, (a) 2-d image from the top (b) Height profile along the red and blue lines indicated in (a), the height of the particles deposited on the silicon substrate is $\sim 15 \mathrm{~nm}$ which is the expected height of TMV particles in an AFM image. 